\title{
Navigation Requirements Development and Performance Assessment of a Martian Ascent Vehicle
}

\author{
Evan J. Anzalone* \\ NASA/Marshall Space Flight Center, Huntsville, AL 35803, USA \\ Hunter Johnston ${ }^{\dagger}$ \\ Texas A\&M University, College Station, TX 77843, USA \\ Jared Leggett, Juan Orphee, Terri Schmitt and Sarah Triana \\ NASA/Marshall Space Flight Center, Huntsville, AL 35803, USA
}

\begin{abstract}
To support development of Martian Ascent Vehicles, analysis tools are needed to support the development of Guidance, Navigation, and Control requirements. This paper presents a focused approach to Navigation analysis to capture development of requirements on initial state knowledge and inertial sensor capabilities. A simulation and analysis framework was used to assess the capability of a range of sensors to operate inertially along a range of launch trajectories. The baseline Martian Ascent Vehicle was used as the input for optimizing a set of trajectories from each launch site. These trajectories were used to perform Monte Carlo analysis dispersing error sensor terms and their effects on integrated vehicle performance. Additionally, this paper provides insight into the use of optical navigation techniques to assess state determination and the potential to use observations of local extraplanetary bodies to estimate state. This paper provides an initial level of performance assessment of navigation components to support continued requirements development of a Martian Ascent Vehicle with applications to both crew and sample return missions.
\end{abstract}

\section{Nomenclature}

$I M U$ = Inertial Measurement Unit
$I N S=$ Inertial Navigation System
$I N U=$ Inertial Navigation Unit
$M A N=$ Martian Ascent Navigation
$M A V=$ Martian Ascent Vehicle

\section{Introduction}

Much work has been done on the architecture development for a large payload Martian Ascent Vehicle. These studies have focused on overall mass to orbit, propulsion systems design, and trajectory design for a notional vehicle. There has not been a detailed assessment of the Guidance, Navigation, and Control (GNC) components required to meet mission requirements. This project intends to address that gap and lay the groundwork for detailed analysis. A Martian craft must be capable of autonomous operations to support initial determination of its launch attitude and location, which are some of the fundamental drivers to insertion accuracy that bound how well the vehicle can attain a desired orbit. The focus of this work is to apply knowledge in Earth-based launch vehicle GNC to a Martian scenario to provide insight into the capabilities afforded by state of the art systems. This helps to identify long lead hardware items and potential risks. The goal of this research is to develop a modeling and simulation environment in order to assess the Navigation system. In order to accomplish this, generic inertial navigation unit models will be integrated into a simulation framework to assess navigation accuracy over a variety of ascent trajectories. This is intended to

\footnotetext{
*PhD, Guidance and Navigation Team Lead, NASA/MSFC/EV42 Guidance, Navigation and Mission Analysis

${ }^{\dagger}$ PhD Student, Intern, NASA/MSFC/EV42 Guidance, Navigation and Mission Analysis Branch
} 
capture launch site sensitivities and cover the wide breadth of potential landing sites, with a focus on how the navigation accuracy affects vehicle sizing in terms of propellant required for on-orbit trajectory corrections.

This paper provides a discussion of the mission and vehicle architecture assumed and provides insight into the trajectory optimization process. The individual tools and analysis approaches are described as well as their results. Sensitivity analysis is provided to highlight key error terms. The system performance under the proposed system is also demonstrated to provide insight for future architecture development and to identify technology gaps and requirements for on-pad state initialization to enable a successful mission. By providing additional insight into this architecture through the application of rigorous launch vehicle GNC design processes, this paper supports future architecture and technology development early in the design process.

In addition to inertial navigation, this study will capture the capabilities and feasibility of using optical systems on the Martian surface to support initialization and in-flight activities. This research seeks to take advantage of star-tracker based navigation methods that are in development to support state determination on Earth in GPS-denied regions. By assessing and understanding the requirement of the navigation architecture, this study will identify possible applications of optical navigation and identify any potential mission constraints that are required for their operation. This will be achieved through documentation of the usage of optical navigation on existing planetary rovers and proposed usages within the terrestrial domain. These capabilities will then also be assessed using the modeling and simulation environment to define their benefits to a Martian ascent navigation architecture.

\section{Background}

As the space industry continues to design and test high mass to orbit launch vehicles, such as the Space Launch System and the Falcon 9 Heavy, the opportunity for a large breadth of planetary missions to the Moon, Mars, and beyond are within grasp. Both government and commercial entities have placed a large focus on future missions to enable a human presence on Mars. In addition to the development of a vehicle to transmit crew and cargo between Earth and Martian systems, architecture and requirements design is ongoing for the vehicle which will be used to land on and return humans from Mars. Much work has been done at the system level to assess key vehicle design parameters, such as Entry, Descent, and Landing technologies to propulsion system design for the ascent vehicle. This paper focuses on a neglected part of the ascent vehicle, the Guidance, Navigation, and Control architecture, in order to understand the capabilities needed and the inherent sensitivities to identify any long lead developments required to meet vehicle requirements on orbital insertion. This works focuses specifically on the Navigation system.

This study builds on the work being performed in ongoing Martian Ascent Vehicles work as documented in [1] to provide additional insight into the Navigation architecture being used. In order to asses this, a simulation environment, built from existing assets, was utilized to simulate an ascent trajectory and assess insertion errors due to the navigation uncertainties. This allows for detailed understanding of the system requirements needed to meet on-orbit trajectory correction maneuver margins. In addition, it seeks to identify gaps in the current architecture well ahead of mission formulation to aid in technology development and identification efforts. In order to provide insight to existing work ongoing in ascent vehicle design, the team coordinated with the AES Martian Ascent Vehicle studies being performed out of the MSFC Advanced Concepts Office, and being led by Tara Polsgrove[1]. This recent analysis provides an overview of a notional vehicle architecture and mission concept. Utilization of this approach provides a mission baseline for trajectory optimization and mission analysis. An overview of the current vehicle design is given below in Figure 1 . 

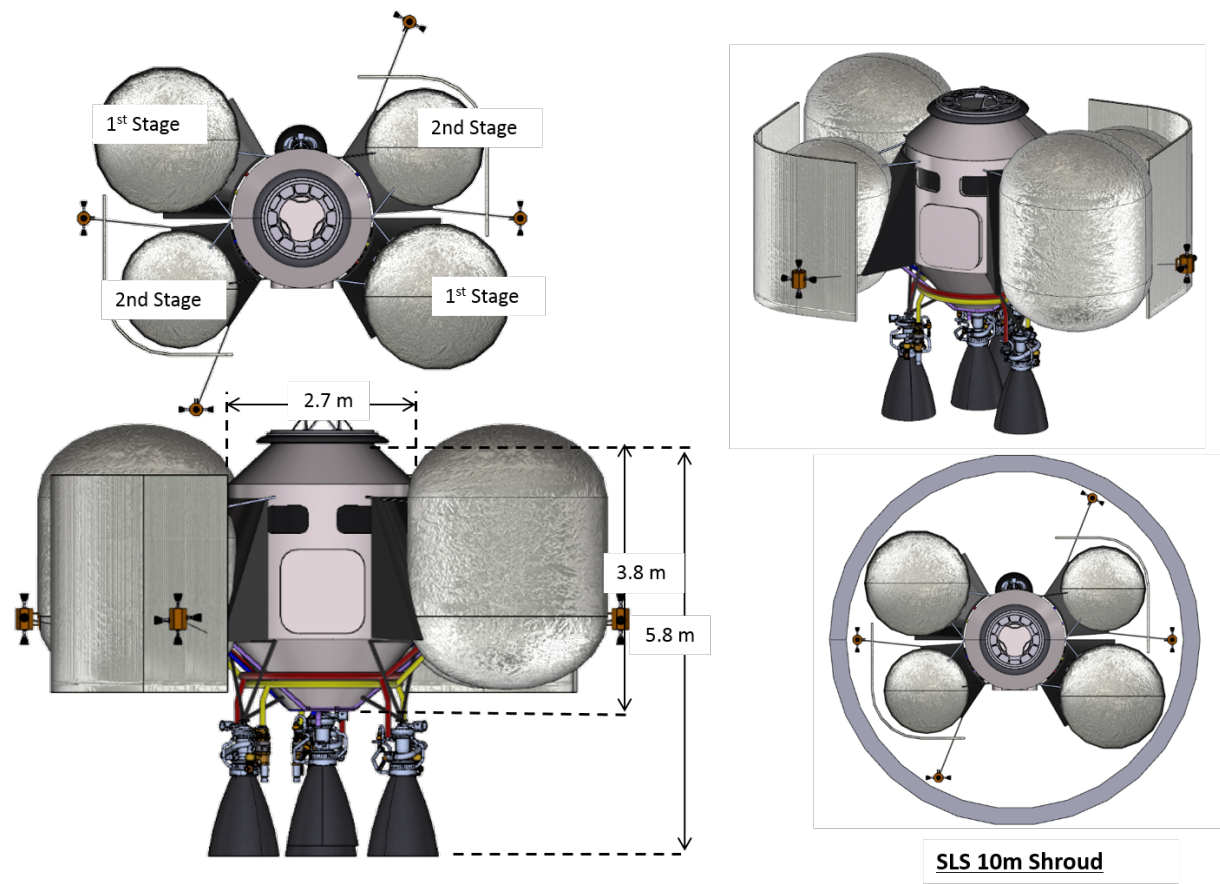

Fig. 1 Notional Martian Ascent Vehicle [1]

The vehicle utilizes two unique stages defined by operation of specific fuel tanks as identified in the conceptual design. As the vehicle continues its ascent trajectory, the tanks identified as "1st Stage" are ejected, leaving the remaining tanks (attached to the reaction control system(RCS) thrusters) to continue the mission into orbit. In order to feed into ascent trajectory optimization, notional masses and mission design parameters (such as staging, engine performance, and vehicle design) are used to provide a high fidelity baseline. Additionally, the overall mission operational timeline in Figure 2 is used to define the orbits and engine firings. The baseline vehicle injects into a $200 \mathrm{~km}$ orbit at an inclination matching the latitude of the launch site. From there, several orbits are completed to enable the vehicle to obtain an Earth-developed state update via the Deep Space Network. Following that, the vehicle executes a series of maneuvers to enter a 1 Martian Day Sun Synchronous orbit to then rendezvous with a Martian Transfer Stage, which will return the crew capsule to Earth. 




Fig. 2 Mission Design

With the mission designed, it is important to understand the current approaches to navigation on planetary surfaces. Most planetary in-situ navigation, outside of Earth, has been focused on Mars exploration rovers. Knowing the rover's position allows engineers and scientists to drive the rover to a location of interest. Currently, Martian rover surface navigation relies on knowing initial position which is then propagated in time along with knowledge of rover attitude, [2-5]. The rover initial position is set to zero, tracking rover position relative to its starting point. To avoid propagation of errors, the starting point gets re-set, usually when science experiments are completed in a given location. In order to determine the rover attitude, the sun vector, obtained using the rover's panoramic camera and on-board ephemeris, plus the Gravity vector, from the on-board IMU, are used. Once the initial rover position and attitude are known, the rover's actual position at a later time is a propagation using the rover's attitude in combination with wheel odometry and visual odometry. More advanced techniques account for the rover's wheel slippage, using an augmented state vector in an Unscented Kalman Filter that combine visual odometry and gyrodometry (gyro rates combined with wheel odometry).

Another rover position determination technique focuses on atmosphere-less bodies, such as the Moon. Celestial observations of the Earth and Sun vectors can be made using a charge-coupled device (CCD) cameras [6] and combined with the local gravity vector to determine rover attitude. Then, with a known initial position, future rover positions are propagated in time using attitude rate gyros and wheel odometry or gyrodometry. Alternatively, star tracker measurements can be used to determine the rover's attitude and use the gravity vector to determine absolute position, without relying on a-priori knowledge of initial position [5, 7, 8]. However, these methods are susceptible to gyro precision and drift. Nonetheless, gyro biases may be corrected with star tracker measurements when the rover is stationary. For a Mars ascent vehicle, optical observations and gyro measurements would be stationary and over long periods of time, minimizing the impact of gyro drift.

Finally, [9] shows another method to determine absolute position. This method takes images of the surface and compares features of interest with an existing database of surface images. This method could provide highly accurate position determination. However, it relies on high resolution surface images and suffice lighting conditions may affect matching algorithms. Future in-situ navigation for a Mars ascent vehicle may rely on a mix of sensed information and a-priori knowledge, including optical observation of celestial bodies and artificial satellites, surface image matching, and 
inertial measurements. Ultimately, launch position accuracy requirements will determine which methods get adopted.

\section{Analysis Approach}

The research's focus is on development of simulation tools to aid in sensitivity and performance analysis for Martian Ascent Vehicles using the notional mission architecture. With these tools in hand, the team is able to analyze system navigation requirements primarily in terms of sensor error budget and initialization knowledge. The goal of this work is to verify the performance capability of proposed light weight sensors and to understand technology gaps in terms of state initialization. In order to asses this, the team is documenting and applying optical navigation methods being developed to support terrestrial navigation in GPS-denied regions through tracking of local satellites, both artificial and natural. An overview of technical objectives of this work are given in Table 1

Table 1 Technical Objectives

\begin{tabular}{c}
\hline Implement array of Gyrocompassing algorithms \\
\hline Implement generic inertial sensor models \\
\hline Implement ascent navigation algorithms \\
\hline Develop ascent trajectories for a family of launch sites \\
\hline Develop tools for in-space assessment of navigation errors \\
\hline Assess feasibility and capability of optical navigation for an ascent vehicle \\
\hline Identify and document algorithms for surface navigation
\end{tabular}

This research effort takes advantage of the team's expertise in ascent navigation architectures for terrestrial vehicles and is transposing those tools into a modular environment for assessment for a wide breadth of planetary missions. This framework includes generic inertial measurement unit sensor errors terms to enable modeling and simulation of a range of capabilities from MEMS-grade to navigation-grade sensors. Additionally, implementation within a modular environment allows for quickly trading initialization algorithms (gyrocompassing), inertial navigation, and environment models, such as gravity. The results of analysis within this framework will support sensor trade studies and requirement development. Additionally, these tools enable assessing the need for optical navigation techniques and their potential benefits.

The Martian Ascent Navigation (MAN) project applies MSFC expertise in launch vehicle GNC and mission design rigor to assess system performance. Using the notional vehicle design, the MAN team generated a series of optimized trajectories using the POST tool[10] for a variety of launch sites. These sites were selected to match the top locations being considered by international collaborators for high value scientific return locations and are given in Table 2 . The ascent trajectories were then used in Monte Carlo analysis of the navigation systems for assessment of system sensitivities and hardware trades. This includes both inertial navigation along the ascent trajectory and state initialization technologies. Several techniques were considered for on-pad alignment, including gyrocompassing and the use of optical systems for both state and attitude estimation. Additionally, sensitivities were generated to understand requirements on understanding initial position on the Martian surface to initialize the navigation algorithms. A variety of inertial measurement units were assessed, from MEMS to navigation-grade instruments, in order to provide insight into mass vs. performance. Additionally, due to the long times between calibration and ascent from Mars, the hardware must be able to maintain calibration for an extended period of time. This part of the analysis provides discussion of the sensitivities to the inertial sensor error terms, algorithms, and state uncertainties in terms of insertion accuracy.

\footnotetext{
*https://mars.nasa.gov/mars2020/mission/timeline/prelaunch/landing-site-selection/
} 
Table 2 Launch Sites Considered in Analysis

\begin{tabular}{|c|c|c|c|c|}
\hline Landing Site & Lat $(\mathrm{degN})$ & Long $(\mathrm{degE})$ & Approx. Elevation $(\mathrm{km})$ & Approx. Buffered Ellipse Axes $(\mathrm{km})$ \\
\hline \hline Colombia Hills & -14.5478 & 175.6255 & -1.93 & $9.6 \times 8.7$ \\
\hline Eberswalde & -23.7749 & -33.5147 & -1.49 & $8.6 \times 7.7$ \\
\hline Holden & -26.6130 & -34.8167 & -2.18 & $9.5 \times 8.1$ \\
\hline Jezero & 18.4386 & 77.5031 & -2.64 & $10.7 \times 8.3$ \\
\hline Mawrth & 23.9685 & -19.0609 & -2.24 & $11.9 \times 9.8$ \\
\hline NE Syrtis & 17.8899 & 77.1599 & -2.04 & $11.1 \times 8.2$ \\
\hline Nili Fossae & 21.0297 & 74.3494 & -0.65 & $9.7 \times 7.7$ \\
\hline SW Melas & -9.8132 & -76.4679 & -1.92 & $9.7 \times 8.7$ \\
\hline
\end{tabular}

In order to tie these state uncertainties to mission-level requirements in terms of delta- $\mathrm{V}$ required to correct for insertion errors, the final states form the ascent analysis are fed into an in-space trajectory design tool, Copernicus, for further analysis. The notional mission design of the MAV is used as a baseline mission scenario to capture the on-orbit operations. Following this mission design, the analysis assumes the craft cruises in Martian orbit for several passes in order to improve its state knowledge via external measurements prior to rendezvous with a transfer stage to return to Earth. Copernicus is used to re-optimize this mission design based on each case's individual insertion errors to assess delta-v deviations and uncertainty form the nominal mission based on the navigation architecture. This allows the sensitivities to be tied to a high level mission metric, mass of the vehicle via delta-V required for in-orbit operations.

This research collaborated with the current Martian Ascent Vehicle architecture to define a baseline for analyzing an ascent trajectory. Initial study results provided this effort with a notional mass budget, propulsion capabilities, and in-space mission design. The team then developed a series of ascent trajectories using the POST ascent trajectory design tool [10] to model a variety of launch sites. The two figures below provide an overview of the optimized trajectories. Figure 3 shows the optimized ascent trajectories from each launch site to insertion into a $200 \mathrm{~km}$ altitude orbit. This figures show the variety of missions being considered and allows the analysis to capture sensitivities and changes in sensor requirements as a function of launch site, principally driving out effects from launch site latitude, which influences gyrocompassing and potential satellite observations. Figure 4 provides a visualization of the ascent trajectories relative to the landing site location. This demonstrates the uniqueness of the ascent trajectories required to meet the desired inclinations.

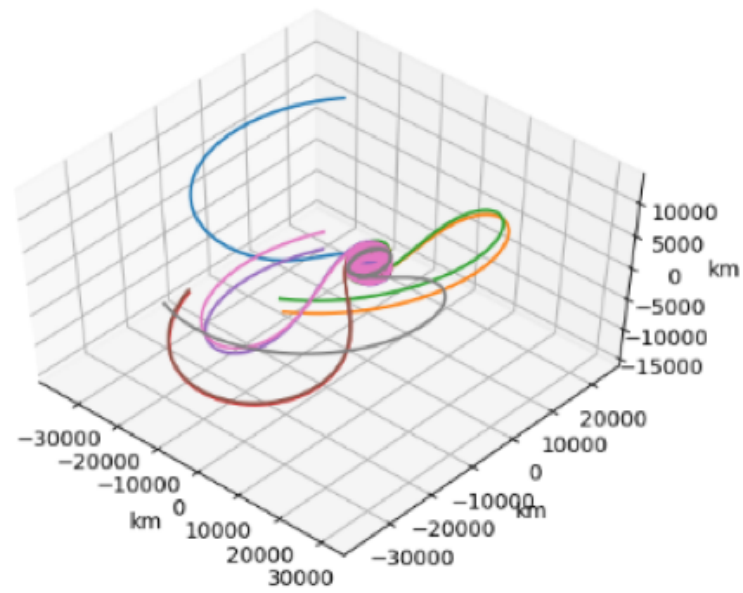

Fig. 3 Optimized Ascent Trajectories into Orbit in Inertial Frame 


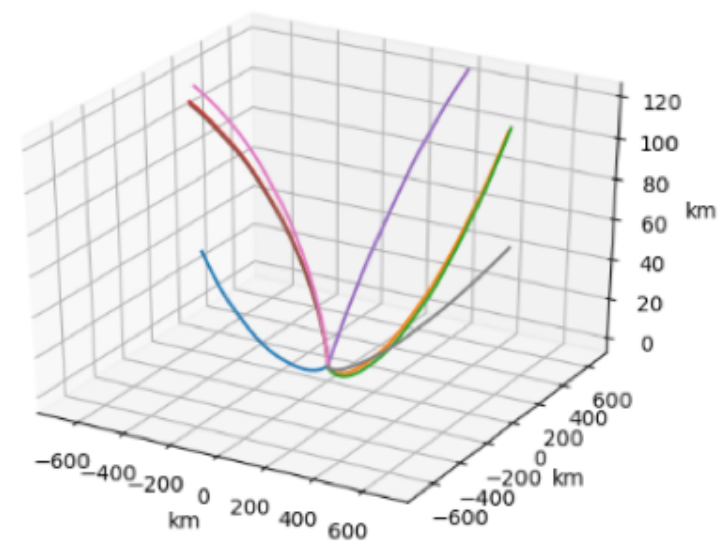

Fig. 4 Trajectories Relative to Landing Site

As part of this research effort, a variety of options for inertial attitude alignment have been implemented into a common framework within a genetic Inertial Navigation Unit model to allow for rapid analysis. The various trades for initial attitude determination are identified in Figure 5. Figure 6 captures the additional trades for understanding the vehicle's initial position on the Martian Surface. The generic model allows for generation of on-pad dynamics on the Martian Surface (allowing for future inclusion of wind modeling and vehicle twist and sway motion), gyrocompassing, and inertial navigation through ascent. This tool allows for sensitivity analysis from sensor specifications to algorithm selection to insertion accuracy.

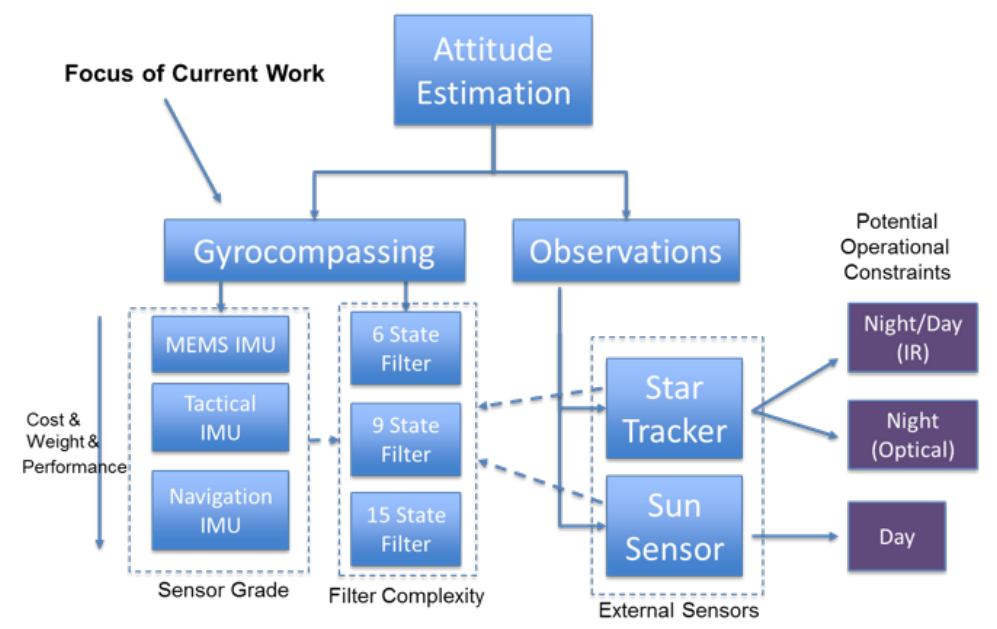

Fig. 5 Attitude Alignment Trades 




Fig. 6 State Initialization Trades

An additional tool was designed to analyze the effort of navigation accuracy on the Martian Ascent Vehicle's requirement to rendezvous with a transfer stage in order to return to Earth. A custom input deck was developed within the Copernicus trajectory optimization tool to re-optimize mission burns in order to meet rendezvous conditions. This links the navigation insertion accuracy to the changes in propellant required to ensure mission success.

\section{Simulation Development}

To support system analysis and requirements development, the Mars Ascent Vehicle Navigation Model (MAN Model) was developed as an object-oriented six degree-of-freedom (6DOF) simulation tool in Python 3. The MAN Model provides standard Monte Carlo simulation and sensitivity analysis capabilities to evaluate a given test case. The model is controlled via an input file, which permits the specification of parameters including but not limited to the path to the reference trajectory file, sensor errors, twist and sway generation controls, and navigation algorithms. On-pad and ascent navigation are supported such that one or both phases may be simulated for a given test case, with gyrocompassing alignment and other navigation filter capabilities available. The MAN Model is a simple to use tool offering 6DOF navigation simulation capabilities in addition to Monte Carlo and sensitivity analysis evaluation functionality.

The MAN Model is implemented in an object-oriented fashion, with the primary objects being instances of the Trajectory and Inertial Navigation System (INS) classes. The Trajectory class is responsible for providing the truth states along the ascent trajectory and generating the on-pad or twist and sway trajectories. The INS class is effectively a wrapper class for the Inertial Measurement Unit (IMU) and Inertial Navigation Unit (INU) classes. The IMU class wraps the Accelerometer and Gyroscope sensor models, which apply sensor noise to the truth input accelerations and rates provided by the Trajectory class and returns the sensed counterparts. The INU class accepts the IMU outputs and integrates them to calculate the navigated states using integration algorithms and navigation filters specified in the input file. During the on-pad mission phase, the INU will perform a gyrocompassing alignment as specified in the input file. The navigated states are subtracted from the corresponding truth states in order to calculate the navigation errors. These data sets can be written to file and or plotted at run time if specified in the input file.

An additional three classes are used to govern the MAN Model: the Planet class, the Twist and Sway Engine (TNS_Engine) class, and the Random Number Generator (RNG) class. The Planet class contains reference data about the body from which the simulated vehicle is launching, including latitude, longitude, altitude, eccentricity, and rotation data, as well as a wrapped SphericalGravity class to act as a gravity model. The TNS_Engine is used to provide twist and sway dispersions to the truth trajectory during the on-pad mission phase similar to that in [11]. The RNG class supplies the start-of-run dispersions to the other classes (e.g. sensor errors) and ensures run repeatability for any test case. Fig. 7 7 shows a diagram of the hierarchy of MAN Model classes and how they interact with each other at run time. The MAN Model also provides the capability to perform sensitivity analyses. This implements a Monte Carlo-driven variance-based approach to determine primary system drivers. The current version of the MAN Model is dependent on 
the Numpy, Scipy, and Pandas modules to handle and manipulate large arrays, read from and write to data files, and provide RNG functionality. The MAN Model also makes use of the Matplotlib module to generate various types of plots to support analysis. Future iterations of the MAN Model may include Cython code to decrease run time.

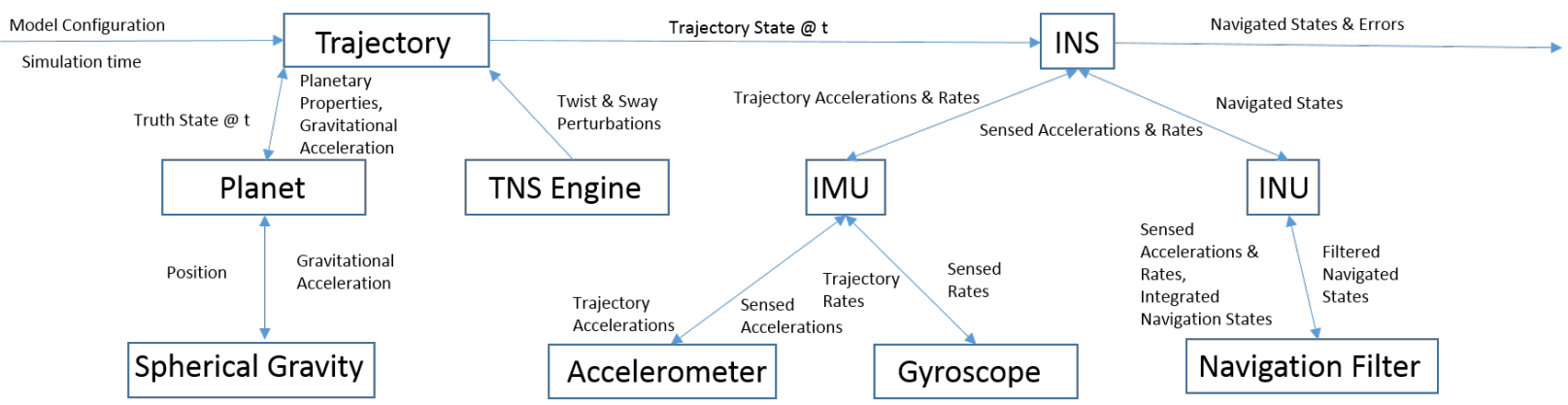

Fig. 7 MAN Model Classes

The MAN Model's internal processes are laid out in Figure 8. First, the parser functions are used to map the configuration parameters in the input file to the Python dictionary. Second, if a sensitivity analysis is specified, the MAN Model generates copies of the input file representing the various combinations of dispersions to be evaluated, saves them to unique directories, and generates a list of the paths to each of these input files. If only a single test case is specified, then this list of input files only contains a path to the original input file. Third, the MAN Model iterates through the list of input files generated in the second step, parsing them sequentially. This does mean that the original input file is parsed twice. The generated Python dictionary is used to configure the MAN Model for each test case, e.g. how many runs to perform or which dispersions to apply. The MAN Model will take advantage of the multiprocessing module to iterate through the specified number of runs before moving on to the next test case, generating new instances of the aforementioned classes at the beginning of each run and saving the results. Fourth, the saved data is reduced to calculate statistical results and generate plots. Fifth, the MAN Model compresses the relevant directories and their contents for archiving.

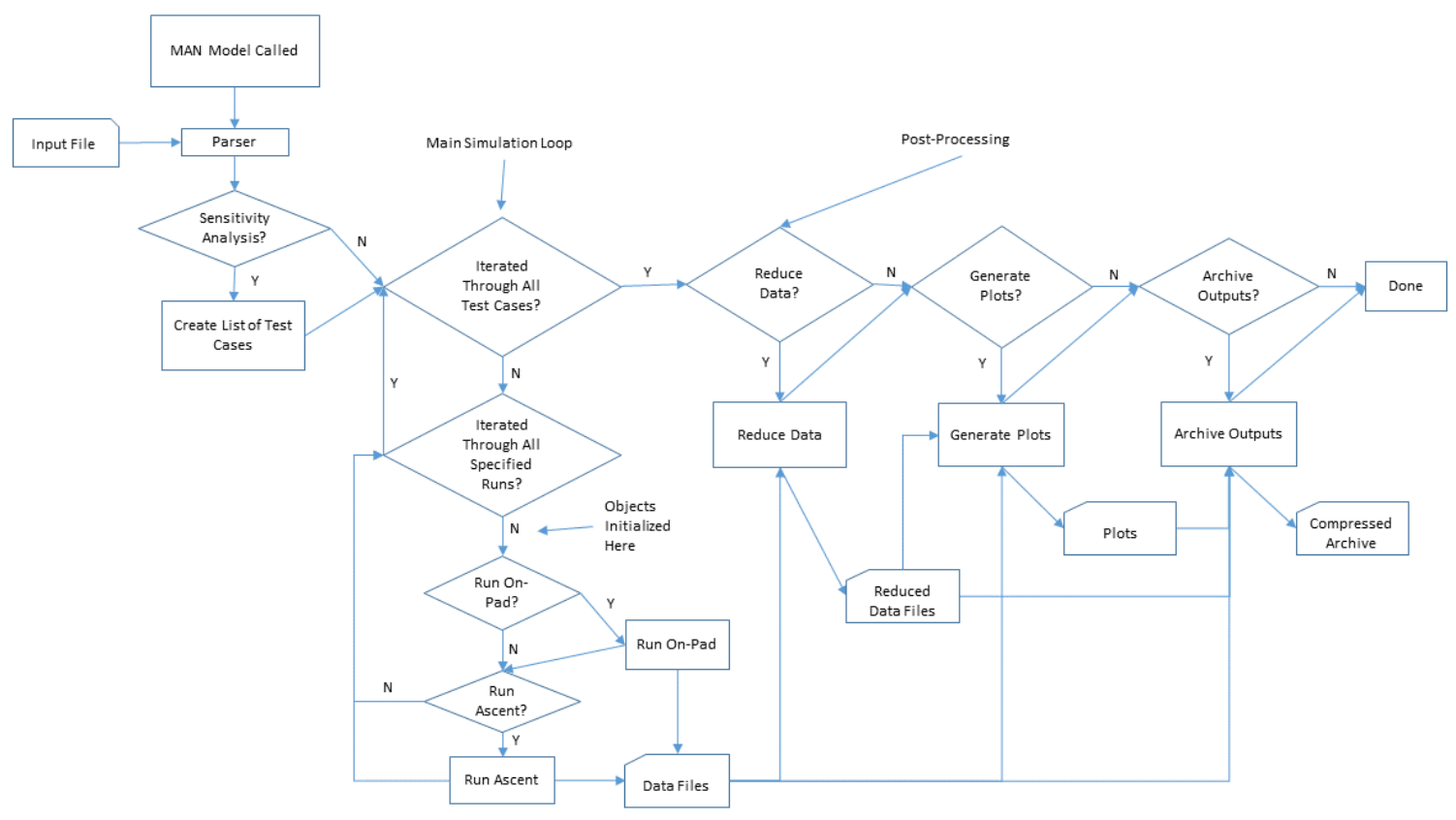

Fig. 8 MAN Model Processes 
The MAN Model is a input file driven, object-oriented, 6DOF simulation tool written in Python 3 developed to support the evaluation of potential Mars Ascent Vehicle navigation system options. It can perform large Monte Carlo studies and sensitivity analyses in addition to automating data reduction and plotting. Due to its object-oriented nature, the MAN Model is easy to modify and new navigation algorithms are simple to implement. The input file structure enables easy comparison of various sensors, launch locations, and trajectories. Because the MAN Model is written in Python, it is portable between systems and can take advantage of built-in multiprocessing to reduce total run time. The MAN Model offers a robust, high fidelity simulation tool that is easy to use for evaluating various navigation systems and algorithms.

\section{Inertial Navigation Performance}

For early missions, any vehicle launching from or landing on Mars must be able to track their state knowledge internally to high precision to meet system requirements with limited external observations. Typically, landing sytems use high grade inertial instruments with altimeters and star trackers providing external measurements along with the proposed use of Terrain Relative Navigation. Other studies have assessed the use of ranging from in-situ orbital assets to support additional knowledge prior to atmospheric entry [12]. The initial part of this analysis focuses on the sole use of inertial instruments to determine initial attitude knowledge and estimate position and velocity over the ascent trajectory. This analysis assesses two components: gyrocompassing and state integration.

\section{A. Inertial Alignment}

For inertial navigation, the system is highly dependent on determination of the vehicle's initial position, velocity, and attitude. Errors in position and velocity directly correlate to insertion error defined as ability to meet desired targeted orbit parameters. Due to the processes involved in strapdown integration[13][14], the system is very sensitive to initial attitude errors. Uncertainties in attitude cause the sensed acceleration to be integrated in the incorrect direction, thus causing increasing position and velocity error. Several approaches exist for inertial sensors to determine their planetary body-relative alignment through a process called gyrocompassing[15]. This processes utilizes measurements of gravity and inertial rotation rate (of the body) to estimate initial attitude.

This analysis focuses on the ability of a range of commercially available IMUs to adequately determine initial attitude. A list of the hardware considered is given in Table 3 . These were selected to provide a mix of both tactical and navigation-grade sensors as well as to compare with requirements analysis for Earth-based launch vehicles. Each of these platforms were assessed to determine their capability to gyrocompass.

Table 3 Assumed Sensor Error Budgets

\begin{tabular}{|l|c|c|c|c|c|}
\hline IMU & $\begin{array}{c}\text { Accel. Bias } \\
\text { ug }\end{array}$ & $\begin{array}{c}\text { Accel. Noise } \\
\text { ug }\end{array}$ & $\begin{array}{c}\text { Gyro Bias } \\
\text { deg/hr }\end{array}$ & $\begin{array}{c}\text { Gyro RW } \\
\text { deg/rt-hr }\end{array}$ & $\begin{array}{c}\text { Gyro Noise) } \\
\text { deg/hr }\end{array}$ \\
\hline \hline HG9900[16] & 25 & & .003 & 0.002 & \\
\hline HQ[17] & 20 & 2 & .003 & 0.001 & 0.001 \\
\hline LN200S[18] & 300 & 35 & 1 & 0.07 & 0.1 \\
\hline MQ[17] & 40 & 4 & .006 & 0.003 & 0.002 \\
\hline HG1930[19] & 5000 & $3 e 5$ & 20 & 0.125 & 1.0 \\
\hline
\end{tabular}

Assuming no external measurements, the analysis focused on the use of coarse alignment algorithms to provide an estimate of the vehicle's initial attitude similar to [11]. The capability of the sensors to execute these algorithms is tightly tied to the ability to measure the vehicle's inertial angular rate while static on the surface. The primary operational variable in this scenario is the amount of data collected prior to state estimation. Coarse alignment algorithms typically work by collecting a large amount of body-frame specific acceleration and inertial measurements averaged over a long period of time to then estimate the alignment between the vehicle and body-centered frame. This is performed to reduce the effect of system noise on the final solution. To asses performance and sensitivity to duration, several Monte Carlos were performed for both duration and sensor specification. The results of the analysis is provided in Table 4 This table 
provides insight into the overall attitude errors. As shown, the ability to determine initial attitude is heavily tied to sensor capability.

Table 4 Coarse Alignment Capability

\begin{tabular}{|c|c|c|c|c|}
\hline IMU & $\begin{array}{c}\text { Time } \\
\text { sec }\end{array}$ & $\begin{array}{c}\text { Level Uncertainty } \\
\text { asec }\end{array}$ & $\begin{array}{c}\text { Level Uncertainty } \\
\text { asec }\end{array}$ & $\begin{array}{c}\text { Azimuth Uncertainty) } \\
\text { asec(deg) }\end{array}$ \\
\hline \hline HG9900 & 120 & $5.09 \mathrm{E}+00$ & $5.11 \mathrm{E}+00$ & $1.78 \mathrm{E}+02(0.05)$ \\
\hline HG9900 & 300 & $5.09 \mathrm{E}+00$ & $5.11 \mathrm{E}+00$ & $1.16 \mathrm{E}+02(0.03)$ \\
\hline HG9900 & 600 & $5.09 \mathrm{E}+00$ & $5.11 \mathrm{E}+00$ & $8.59 \mathrm{E}+01(0.02)$ \\
\hline HQ & 120 & $9.74 \mathrm{E}+00$ & $9.32 \mathrm{E}+00$ & $3.28 \mathrm{E}+02(0.09)$ \\
\hline HQ & 300 & $1.46 \mathrm{E}+01$ & $1.36 \mathrm{E}+01$ & $5.17 \mathrm{E}+02(0.14)$ \\
\hline HQ & 600 & $2.77 \mathrm{E}+01$ & $2.88 \mathrm{E}+01$ & $7.22 \mathrm{E}+02(0.20)$ \\
\hline LN200S & 120 & $6.52 \mathrm{E}+01$ & $6.52 \mathrm{E}+01$ & $3.62 \mathrm{E}+04(10.06)$ \\
\hline LN200S & 300 & $6.71 \mathrm{E}+01$ & $6.57 \mathrm{E}+01$ & $5.57 \mathrm{E}+04(15.48)$ \\
\hline LN200S & 600 & $6.55 \mathrm{E}+01$ & $6.50 \mathrm{E}+01$ & $8.12 \mathrm{E}+04(22.55)$ \\
\hline MQ & 120 & $2.13 \mathrm{E}+01$ & $2.06 \mathrm{E}+01$ & $6.88 \mathrm{E}+02(0.19)$ \\
\hline MQ & 300 & $3.01 \mathrm{E}+01$ & $2.85 \mathrm{E}+01$ & $1.04 \mathrm{E}+03(0.29)$ \\
\hline MQ & 600 & $4.02 \mathrm{E}+01$ & $3.88 \mathrm{E}+01$ & $1.44 \mathrm{E}+03(0.40)$ \\
\hline HG1930 & 120 & $1.75 \mathrm{E}+05$ & $2.11 \mathrm{E}+05$ & $1.98 \mathrm{E}+05(54.93)$ \\
\hline HG1930 & 300 & $1.85 \mathrm{E}+05$ & $2.15 \mathrm{E}+05$ & $1.98 \mathrm{E}+05(55.04)$ \\
\hline HG1930 & 600 & $1.90 \mathrm{E}+05$ & $2.15 \mathrm{E}+05$ & $1.99 \mathrm{E}+05(55.34)$ \\
\hline
\end{tabular}

For coarse alignment, the initial estimate is expected to improve as a function of time up to the limit of sensor bias and random walk specifications, as well as latitude of the landing site. The results above do not match this behavior, primarily due to the limited number of cases ran. The results provide insight into the capabilty of inertial alignment based on sensor grade. As seen, navigation grade units can do this very well (within 100s of arcsecs) but as the noise terms increase, so too does the attitude error. For example, the tactical MEMS sensor exhibits very large uncertainty. As given in [20], it is possible to determine the best possible gyrocompassing accuracy as a function of launch latitude, planetary body inertial rate, and the sensor's angular random walk specification (primary drivers of system performance). In order to capture long-term behavior, the launch sites and sensor specs were assessed. The results are shown in Table 5. This again shows the breadth of capability over sensor performance. This analysis is specific to the Martian surface and launch sites, considering local latitude and Martian inertial rate.

Table 5 Gyrocompassing Launch Site and Noise Sensitivity Attitude Error Uncertainty (arcseconds)

\begin{tabular}{|c|c|c|c|c|c|c|c|c|}
\hline IMU & Colombia Hills & Eberswalde & Holden & Jezero & Mawrth & NESyrtis & NiliFossae & SWMeleas \\
\hline \hline LN200S & 719.5 & 761.0 & 779.0 & 734.1 & 762.2 & 731.8 & 746.1 & 706.8 \\
\hline HG9900 & 20.6 & 21.7 & 22.3 & 21.0 & 21.8 & 20.9 & 21.3 & 20.2 \\
\hline HG1930 & 1284.8 & 1358.9 & 1391.0 & 1310.9 & 1361.0 & 1306.8 & 1332.4 & 1262.1 \\
\hline MQ & 30.8 & 32.6 & 33.3 & 31.5 & 32.7 & 31.4 & 32.0 & 30.3 \\
\hline HQ & 10.5 & 11.1 & 11.4 & 10.7 & 11.1 & 10.7 & 10.9 & 10.3 \\
\hline
\end{tabular}

While more complex methods can be used to gyrocompass, such as Kalman Filtering techniques, this analysis still provides insight into the capabilities of the hardware platforms to determine initial attitude. This knowledge will be 
used to feed vehicle-level trades in terms of hardware selection and operational architecture. Additionally, these results will be used to help inform selections that must balance performance and sensor SWAP. These platforms shown in Table 3 represent not just changes in performance, but also in terms of mass, with the navigation grade units typically much higher and requiring more electrical power than smaller MEMs or tactical-grade systems. The following section focuses on the capability to navigate over an ascent trajectory.

\section{B. Ascent Performance}

Once the vehicle has knowledge of its initial position and attitude, it is possible to use body-frame measured specific acceleration and inertial angular rate to integrate its state estimate forward in time. In addition to assessing sensitivity to specific error terms, this analysis also included a range of launch location as laid out in Table 2 In these cases, a Monte Carlo analysis of 500 cases was performed for each IMU considered for each trajectory. Though the sample size shown here in this analysis is small, the results are intended to provide insight to focus more detailed and intensive studies. The data in Table 6 shows position, velocity, and attitude errors in terms of mean and one sigma uncertainty for each launch site for each IMU. This analysis clearly shows the strong benefit of navigation-grade units and weak performance of lower grade instruments. Additionally, in terms of pure inertial performance, the results are fairly independent of the launch site and the optimized trajectory. This is primarily due to the similar profiles of the launch profile, with no vehicles exhibiting dynamics significantly different from the others.

Table 6 Navigation Errors at Insertion per Launch Site and IMU

\begin{tabular}{|c|c|c|c|c|c|}
\hline \multirow{2}{*}{$\begin{array}{c}\text { Launch } \\
\text { Site }\end{array}$} & IMU & \multicolumn{2}{c|}{ Position Error $(\mathrm{m})$} & \multicolumn{2}{c|}{ Velocity Error $(\mathrm{m} / \mathrm{s})$} \\
\hline \hline Colombia Hills & HG9900 & 146.9 & 61.5 & 0.6 & 0.3 \\
\hline Colombia Hills & HQ & 1439.8 & 616.6 & 8.0 & 3.4 \\
\hline Colombia Hills & LN200S & 9451.6 & 4904.1 & 68.2 & 35.0 \\
\hline Colombia Hills & MQ & 2881.9 & 1230.6 & 16.0 & 6.8 \\
\hline Colombia Hills & HG1930 & $2.1 \mathrm{E}+08$ & $9.0 \mathrm{E}+07$ & $1.2 \mathrm{E}+06$ & $5.0 \mathrm{E}+05$ \\
\hline \hline Eberswalde & HG9900 & 146.3 & 65.1 & 0.6 & 0.3 \\
\hline Eberswalde & HQ & 1437.5 & 611.7 & 8.0 & 3.4 \\
\hline Eberswalde & LN200S & 9612.2 & 5029.1 & 69.0 & 36.8 \\
\hline Eberswalde & MQ & 2874.7 & 1225.7 & 15.9 & 6.7 \\
\hline Eberswalde & HG1930 & $2.1 \mathrm{E}+08$ & $9.0 \mathrm{E}+07$ & $1.2 \mathrm{E}+06$ & $5.0 \mathrm{E}+05$ \\
\hline \hline Holden & HG9900 & 146.8 & 65.5 & 0.6 & 0.3 \\
\hline Holden & HQ & 1444.2 & 614.4 & 8.0 & 3.4 \\
\hline Holden & LN200S & 9690.7 & 5071.7 & 69.6 & 37.1 \\
\hline Holden & MQ & 2888.0 & 1230.9 & 16.0 & 6.7 \\
\hline Holden & HG1930 & $2.1 \mathrm{E}+08$ & $9.1 \mathrm{E}+07$ & $1.2 \mathrm{E}+06$ & $5.0 \mathrm{E}+05$ \\
\hline \hline Jezero & HG9900 & 151.7 & 62.6 & 0.6 & 0.3 \\
\hline Jezero & HQ & 1455.4 & 635.2 & 8.0 & 3.5 \\
\hline Jezero & LN200S & 9331.9 & 4959.6 & 67.7 & 35.7 \\
\hline Jezero & MQ & 2878.2 & 1239.6 & 16.0 & 6.9 \\
\hline Jezero & HG193 & $02.1 \mathrm{E}+08$ & $9.0 \mathrm{E}+07$ & $1.2 \mathrm{E}+06$ & $5.0 \mathrm{E}+05$ \\
\hline
\end{tabular}

Fig. 9 provides more insight into the dispersion between medium and high quality IMU platforms. Each image shows a plot of fifty cases. This shows the position errors across the trajectory for a fixed launch trajectory. These diagrams are typically used to identify outlaying cases and provide insight into bulk statistics. As seen, the dispersions 
reduce as a function of sensor capabilities.
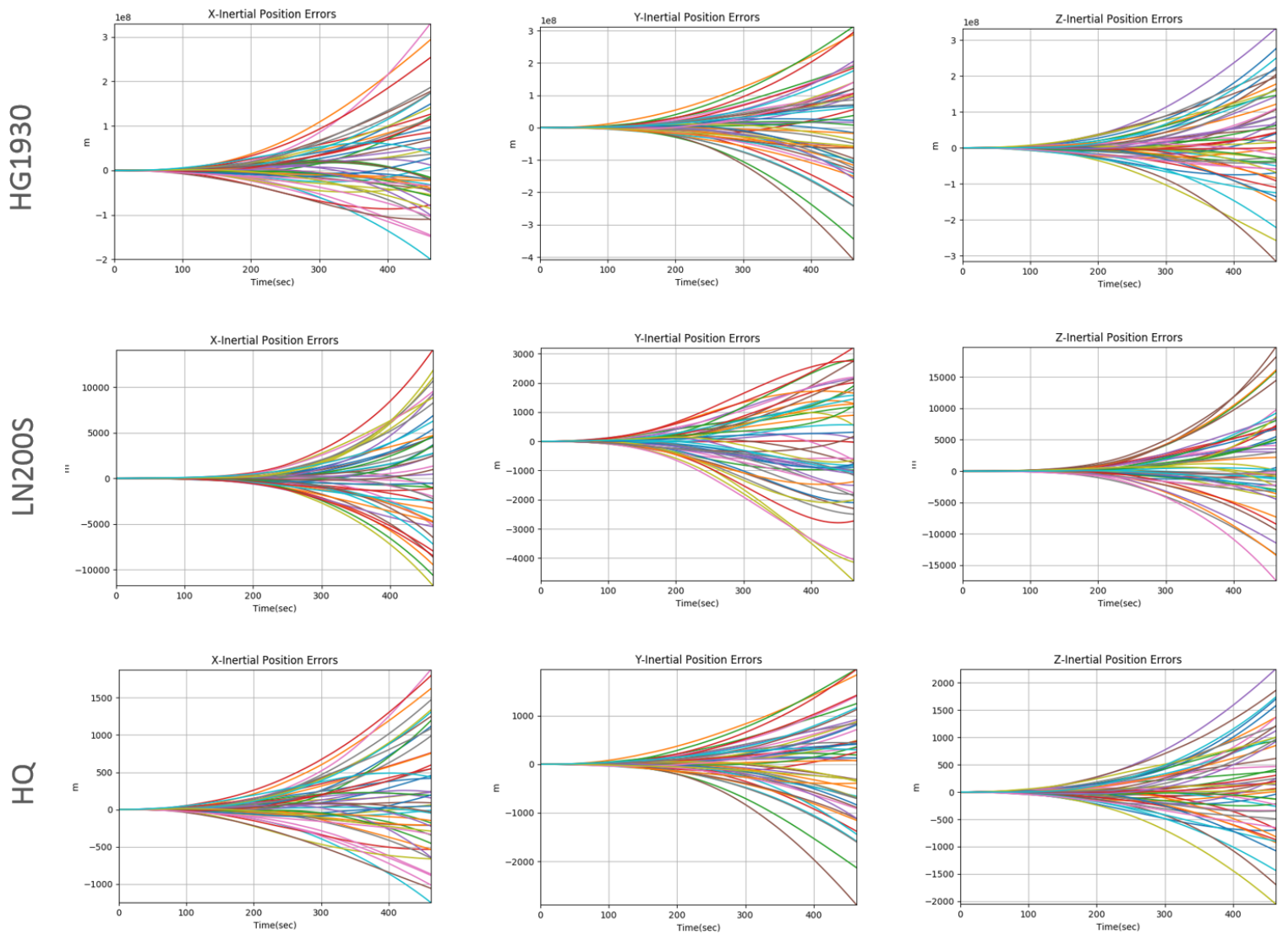

Fig. 9 Ascent Inertial Navigation Insertion Errors (m)

These results assumed perfect initial knowledge to focus on comparisons between different sensors. This also provides insight to decouple initial attitude uncertainty from insertion error. In order to provide insight into this effect, a sensitivity analysis was performed to understand this behavior. A perfect IMU was assumed and Monte Carlos of 500 runs were performed to capture errors at the end of the ascent trajectory. A range of initial attitude errors were modeled and applied at the initial conditions and run along the ascent trajectory. These errors were modeled as total attitude uncertainty and applied as an error quaternion to initial attitude with a normal dispersion on total angle about a random rotation vector. The normalized vector and angle was then used to form a quaternion and applied to the initial attitude. Table 7 shows the results from this analysis. 
Table 7 Insertion Performance as a Function of Initial Attitude Error

\begin{tabular}{|c|c|c|c|c|}
\hline $\begin{array}{c}\text { Attitude Error } \\
\text { Uncertainty } \\
(\mathrm{deg})\end{array}$ & $\begin{array}{c}\text { Position Error } \\
\text { Mean } \\
(\mathrm{m})\end{array}$ & $\begin{array}{c}\text { Position Error } \\
\text { Uncertainty } \\
(\mathrm{m})\end{array}$ & $\begin{array}{c}\text { Velocity Error } \\
\text { Mean } \\
(\mathrm{m} / \mathrm{s})\end{array}$ & $\begin{array}{c}\text { Velocity Error } \\
\text { Uncertainty } \\
(\mathrm{m} / \mathrm{s})\end{array}$ \\
\hline \hline 10 & 186031.99 & 153734.70 & 802.36 & 654.48 \\
\hline 1 & 18794.17 & 15725.01 & 81.02 & 66.80 \\
\hline 0.1 & 1880.03 & 1572.75 & 8.10 & 6.68 \\
\hline 0.01 & 190.07 & 156.41 & 0.81 & 0.67 \\
\hline 0.001 & 27.20 & 13.37 & 0.08 & 0.07 \\
\hline
\end{tabular}

As seen in these results, the baseline capability of the insertion performance of the various sensors are limited by the initial attitude error. The analysis shows that an order of magnitude increase in attitude uncertainty amount to an order of magnitude increase in insertion errors. The statistics were generated by taking the root-sum-square position and velocity at the end of each ascent trajectory. These data sets were then used to asses mean and 1-sigma uncertainty on the errors. This data shows the sensitivity to initial alignment for navigation, important in both openand closed-loop guidance algorithms. This data can be tied back to the gyrocompassing results to understand at a baseline level what the IMUs are capable of. The only caveat is that insertion errors and gyrocompassing errors are directly correlated and though the results in Table 7 assume a perfect sensor, the resulting error with a real sensor will be much larger, due to error such as scale factor and bias integrated over the ascent trajectory. One result this ties into is the potential need for an optical attitude sensor (i.e. star tracker or fine sun sensor) in order to decouple initial attitude knowledge from insertion performance. This could enable using lower grade sensors to meet required insertion performance. Additionally, more complex gyrocompassing algorithms could also be used to attempt to further improve initial alignment and thus insertion performance.

\section{In-space Performance Evaluation}

In order to tie the insertion uncertainty to vehicle-level performance metrics, in-space analysis is used. To support this project, a delta-delta-v analysis was set up similarly to that done in [21]. In this analysis approach, state uncertainties in vehicle insertion conditions are assessed to determine their effect on the overall mission. This is completed by re-optimizing the in-space mission design based on the dispersed states. The difference in overall delta- $\mathrm{V}$ required to meet final conditions (i.e. insertion into the desired 1 Martian Sol orbit) provides a measure of the sensitivity due to navigation errors. Any differences from the nominal mission profiles can thus be attributed to navigation errors. This data is captured through re-optimization due to the nonlinear complex behavior in the orbital environment and complex mission design as shown above in Fig. 2 . This scenario would work operationally through the assumption of an external high accuracy state measurement to provide detailed state knowledge prior to any in-orbit burn planning and execution.

In order to simulate this within the MAV architecture, the notional mission design was implemented into the Copernicus trajectory optimization tool[22]. This implementation of the mission was then compared back to the trajectories generated by the ascent tool, POST, for verification. A Python script was developed to pull in each dispersed insertion state and re-optimize the trajectory in Copernicus. The re-optimization followed the nominal mission objectives: to minimize delta- $\mathrm{V}$, and to meet the nominal mission plan and final orbit conditions. The difference in the delta-V used in the nominal mission and the delta-V used in the "off-nominal" gives us our delta-delta-V. For this analysis, a Monte Carlo-based sensitivity approach was used to create sets of states with given uncertainties in position and velocity to be assessed within Copernicus to provide initial insight into the design space. 


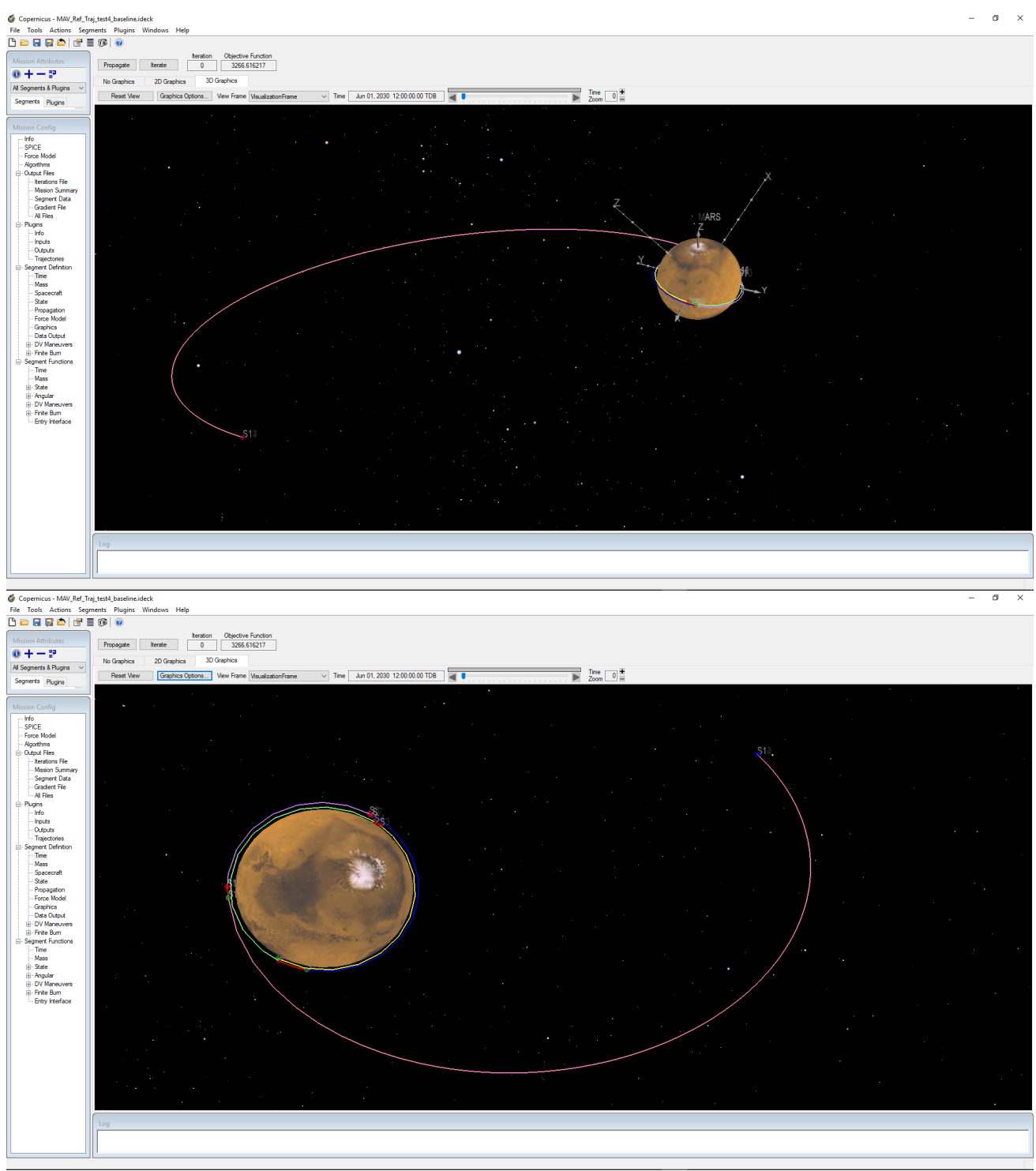

Fig. 10 Views in Copernicus of the Notional Mission

As stated above, this analysis consisted of a Monte Carlo analysis on states at insertion to re-assess its affect on overall mission requirements. For this, position uncertainties of $0.1,1.0,10.0$, and $100 \mathrm{~km} 1$-sigma were used with velocity uncertainties of $0.01,0.1$, and $1 \mathrm{~km} / \mathrm{sec}$. This was intended to capture a range of insertion capability. Every permutation of position and velocity uncertainty from these metrics was used to generate 100 dispersed states at the end of the nominal trajectory at the initial insertion into Martian orbit. Each case was then used to seed the Copernicus simulation to assess performance of a re-optimization to capture changes in delta-velocity required. The results are shown below in Table 8 . As seen, there is a correlation to both increasing position and velocity state, with velocity being a key driver. Additionally, the correction values can be seen to be fairly small, within a $\mathrm{m} / \mathrm{s}$. This is due to the extended mission timeline and loose constraints on time of entering into the one sol orbit. With the long duration, small changes in velocity are able to propagate to large changes in position to meet desired orbital conditions. Additional constraints on meeting intermediate orbits and specifying time of targeted state would cause these values to increase. 
Table 8 Delta-Delta-Velocity(m/s) Due to Insertion Errors

\begin{tabular}{|c|c|c|c|c|}
\hline Velocity 1-Sigma(km/sec) & \multicolumn{4}{|c|}{ Position 1-Sigma(km) } \\
\hline \hline & 0.1 & 1 & 10 & 100 \\
\hline 0.01 & 0.010 & 0.009 & 0.013 & 0.066 \\
\hline 0.1 & 0.063 & 0.078 & 0.080 & 0.111 \\
\hline 1 & 0.287 & 0.221 & 0.240 & 0.301 \\
\hline
\end{tabular}

This work assumed a closed loop guidance solution that directly ties navigation state into guided state. Using an open loop trajectory could allow the use of lower grade sensors but at the cost of increased insertion uncertainty. Additionally, environment effects and other state uncertainties must be well characterized to analyze the robustness of the optimized trajectory. Future work on requirements assessment will assess both open- and closed-loop guidance techniques to continue assess the effect on vehicle-level requirements.

Additional future work in this area is to continue to mature the Copernicus input deck and the POST ascent trajectories as the vehicle architecture matures. Additionally, the individual runs performed above in the assessment of inertial navigation errors capture a range of the errors produced by the navigation sensors. The future integration of this tool with the overarching simulation architecture will enable Monte Carlo analysis from state initialization through ascent and finally in-orbit operations to assess a complete vehicle. This will allow linking the initial system uncertainties to integrated vehicle metrics at a mission level.

\section{Application of Optical Navigation Techniques to State Initialization}

The capability of inertial navigation indicates a potential need for alternate approaches to state initialization. The first stage of the mission, or the pre-launch phase, presents a unique situation. While the MAV is not inertially fixed, the position is constrained to the Martian surface, and is not changing in a Mars Centered Mars Fixed (MCMF) reference frame. Since the MAV's motion is related to the rotation of Mars (which is know with high accuracy) the problem can be simplified. A map between all the relevant coordinate frames and the information needed to define these rotations is provided in Fig. 11. It can be seen that rotation $C_{B I}$ is determined using a star tracker, while the rotation $C_{B L}^{\mathrm{T}}$ is determined by an inclinometer array.



Fig. 11 Direction Cosine Matrix map between inertial frame and sensor-body frame (sometimes referred to as sensor frame)

\section{A. Stellar Positioning System}

A prior study funded by NASA/MSFC called the Stellar Positioning System (SPS) [23, 24], looked to use the relationship described in Fig. 11 to relate the attitude of the spacecraft acquired by a star tracker to the position by way of inclinometers to determine the spacecraft's orientation in the local reference frame. Preliminary results were obtained 
from this method and accuracy was determine to be within 40 meters for Earth-based measurements. For this technique, the position estimation is a function of error in the measurement of the gravity direction, attitude error, and the size of the body the observer is on. To fully understand these implications for Mars-based observation a simulation was developed and verified with the SPS hardware test. From this, a Monte Carlo analysis was conducted varying gravity measurement error and attitude error to determine the expected error in position estimation.

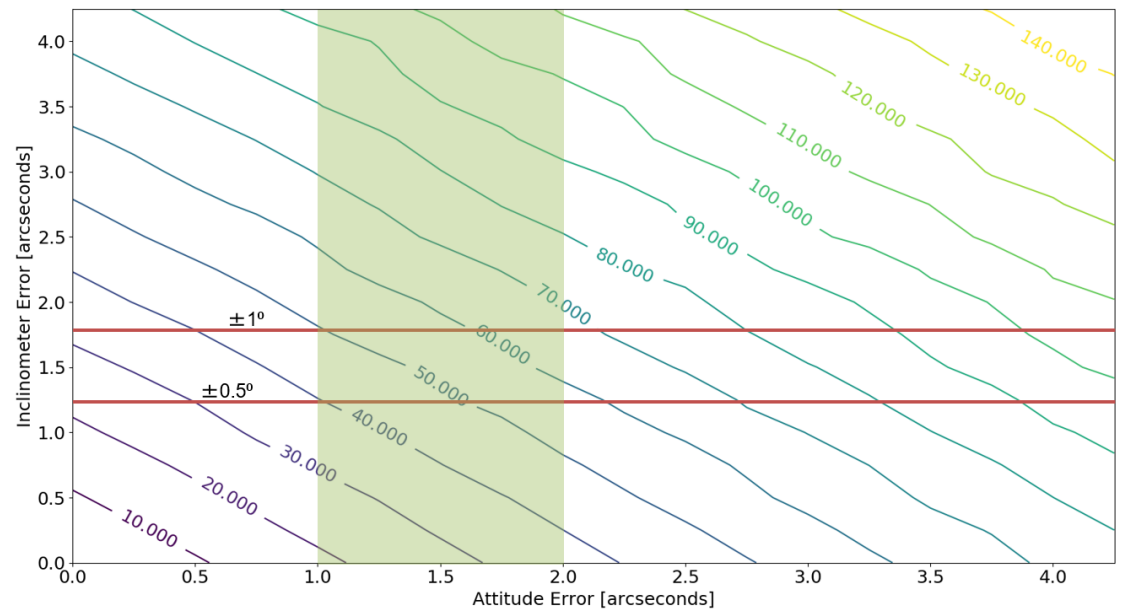

Fig. 12 SPS sensitivity analysis of position estimation related to both star tracker and inclinometer accuracy. The contour lines represent the maximum position error of a 1000 trial Monte Carlo simulation.

In Fig. 12, the maximum position error for the two parameters is detailed. In this, the level curves represent maximum position error in meters. Additionally, the green highlighted area represents the attitude after the Kalman filter convergence and the red horizontal lines show the range of specific inclinometers for both $\pm 0.5^{\circ}$ and $\pm 1^{\circ}$. The same test was captured in Fig. 13, except the level curves detail the average position error in meters.



Fig. 13 SPS sensitivity analysis of position estimation related to both star tracker and inclinometer accuracy. The contour lines represent the average position error of a 1000 trial Monte Carlo simulation.

\section{B. Single Satellite Observation}

In addition to the study in the Stellar Position System, a technique was developed for position estimation based on single satellite observations. It was determined that the major factors in determining position of the spacecraft are 
attitude accuracy and angular resolution of optics, two Monte Carlo tests were constructed to analyze the sensitivity of these two quantities along with the distance to the orbiting satellite. First, Fig. 14 describes the relationship between angular resolution and distance to the satellite.

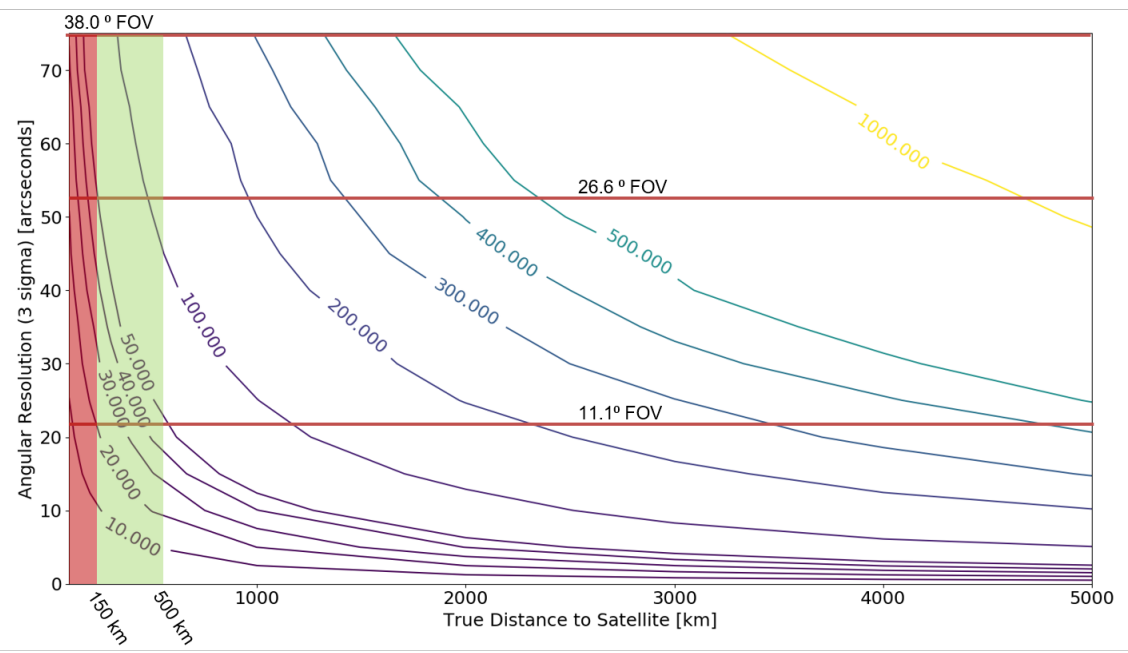

Fig. 14 Effects of angular resolution of optics and distance to satellite on accuracy of position estimation. The level curves represent the average error in meters.

In this figure, the red band signifies distances smaller than any current Martian satellites and must not be considered in the analysis of the graph. Furthermore, the green band is the expected altitude of most Martian satellites based on their periareion and apoareion. It must be noted that the periareion is a firm lower limit, but the apoareion is only valid if the satellite is directly over head. Therefore, it can be expected that observing a satellite from the Martian surface ranges may exceed $500 \mathrm{~km}$. Additionally, the different cameras used in this study are highlighted by the red horizontal lines and these define their angular resolution. It can also be seen from this graph that both Phobos and Deimos are beyond the range of this graph and even with a perfect centroiding algorithm, the error in distance due to angular resolution removes them as a possibility for accurate ground based position estimation. It can be seen that for a single measurement, the expected error ranges from 10 - 100 meters.

Next, the other major parameter effecting position estimation is spacecraft estimated attitude. An offset in the sensors relationship to the inertial frame will introduce error that will add onto the error produced from angular resolution. Fig. 15 details the expected error due to attitude estimation. 




Fig. 15 Effects of spacecraft attitude estimation and satellite distance on accuracy of position estimation. The level curves represent the average error in meters.

Again, in this figure the red area represents lower altitudes than all current Martian assets, with the vertical green region the same as Fig. 14. Furthermore, the horizontal green region displays a range of expected attitude error of 1 - 2 arcseconds which was the average error observed after Kalman filter convergence. It can be seen that $1-2$ arcseconds of attitude error contributes to about 10 - 40 meters of position error.

\section{Conclusions}

This research provides insight into the Navigation architecture and its requirements as part of a Martian Ascent Vehicle. As part of the work, detailed simulation tools were developed to enable a wide variety of analysis. The team assessed inertial attitude determination capability using both traditional strapdown approaches as well as aiding with a star sensor. The use of the star sensor places operational limits on the launch time to enable sensor functionality. Several grades of inertial measurements were then assessed to understand capability amongst them in terms of navigation capability. Although the analysis used an open loop trajectory, the use of closed-loop guidance algorithms will tie the navigation uncertainty directly into insertion error. As such, the results provide initial information into the development of requirements of the navigation system and their link to vehicle-level requirements. As the navigation state is coupled into the as-flown trajectory with integrated Guidance and Control, it is expected that the sensitivity to these errors will grow more important.

The inertial navigation results identify the initial state, position, and attitude as important drivers of vehicle insertion performance. Research into optical navigation techniques has provided a capable solution to both state and attitude determination. This capability, though, places additional constraints on the onboard timing knowledge of the vehicle prior to initialization. While this research attempted to apply additional optical navigation techniques to determine initial position using observations of local planetary bodies and satellites, the results show limited functionality with the current state of the art in optical sensor hardware. This is due to their limited number, requirements on highly accurate state predictions, and their high altitude orbits. Future work will continue to investigate filtering approaches to improve this capability.

These results provide insight into the navigation requirements for a Martian Ascent Vehicle. While the inertial and guidance techniques may be similar to typical launch vehicle approaches, the drive to reduce system size and ability to function with well aged calibrations provide additional constraints to the system. These include the need for detailed analysis and development of systems to initialize position and velocity on the pad. In order to ensure a robust design, these elements should be considered early in concept design. 


\section{Forward Work}

These tools will be integrated into one simulation framework to allow for end-to-end analysis to enable assessment and documentation of the systems performance and requirements. The models will be used to document and support Martian Ascent Vehicle navigation system design trades and to help identify potential long lead items and risks to mission success. With this environment in place, it will allow for additional detail into system performance assessment and can be wrapped into full GNC simulations of the vehicle design. Similarly, this work will help to identify areas of opportunity for optical navigation in planetary ascent vehicles, providing rationale for continued studies into their application and capabilities.

Ongoing studies will continues to assess the potential performance gains enabled by a Kalman Filter-based Gyrcompassing algorithm. Additionally, the team will assess the potential to integrated the optical navigation state estimates into the alignment filter to identify any gains. With the framework in place, the team will also be able to complete detailed sensitivity analysis on the navigation architecture to continue assessment of the algorithms and sensor grade selected.

Future work in this area will include continued sensitivity analysis to support vehicle architecture designs. With the navigation being assessed against an open-loop trajectory, the next step is to implement closed-loop guidance and control to meet the optimized insertion targets. The team is currently working to implement Optimal Guidance algorithms similar to [25] to begin to assess guidance design and sensitivities, particularly due to local environments and winds at launch. This will require a model to capture control response to feed into RCS or Thrust Vector Control design. This will enable the team to identify long lead architectures in terms of integrated Guidance and Navigation performance as well as on pad remote autonomous operations. Additionally, the team is continuing forward work assessing hardware platforms to test and demonstrate the initial position estimation algorithms and characterize sensor gyrocompassing capability.

\section{Acknowledgments}

The team must acknowledge the work of the MSFC Advanced Concepts Office and the Advanced Explorations Systems group, in particular Tara Polsgrove, for their support and work on the development of the Martian Ascent Vehicle architecture and providing the data needed to seed and provide a baseline for this analysis. The team is also grateful to the project office for their funding of this work.

\section{References}

[1] Polsgrove, T. P., Thomas, H. D., Sutherlin, S., Stephens, W., and Rucker, M., "Mars Ascent Vehicle Design for Human Exploration," AIAA SPACE 2015, 2015.

[2] Sakai, A., Tamura, Y., and Kuroda, Y., "An efficient solution to 6DOF localization using Unscented Kalman Filter for planetary rovers," 2009 IEEE/RSJ International Conference on Intelligent Robots and Systems, 2009, pp. 4154-4159. doi:10.1109/IROS.2009.5354677.

[3] Ali, K. S., Vanelli, C. A., Biesiadecki, J. J., Maimone, M. W., Cheng, Y., Martin, A. M. S., and Alexander, J. W., "Attitude and position estimation on the Mars exploration rovers," 2005 IEEE International Conference on Systems, Man and Cybernetics, Vol. 1, 2005, pp. 20-27 Vol. 1. doi:10.1109/ICSMC.2005.1571116.

[4] Furgale, P., Enright, J., and Barfoot, T., "Sun Sensor Navigation for Planetary Rovers: Theory and Field Testing," IEEE Transactions on Aerospace and Electronic Systems, Vol. 47, No. 3, 2011, pp. 1631-1647. doi:10.1109/TAES.2011.5937255.

[5] Enright, J., Barfoot, T., and Soto, M., "Star tracking for planetary rovers," 2012 IEEE Aerospace Conference, 2012, pp. 1-13. doi:10.1109/AERO.2012.6187042.

[6] Pingyuan, C., Fuzhan, Y., and Hutao, C., "Attitude and position determination scheme of lunar rovers basing on the celestial vectors observation,” Integration Technology, 2007. ICIT'07. IEEE International Conference on, IEEE, 2007, pp. 538-543.

[7] Sigel, D. A., and Wettergreen, D., "Star tracker celestial localization system for a lunar rover," 2007 IEEE/RSJ International Conference on Intelligent Robots and Systems, 2007, pp. 2851-2856. doi:10.1109/IROS.2007.4399510.

[8] Ning, X., and Liu, L., "A Two-Mode INS/CNS Navigation Method for Lunar Rovers," IEEE Transactions on Instrumentation and Measurement, Vol. 63, No. 9, 2014, pp. 2170-2179. doi:10.1109/TIM.2014.2307972. 
[9] Boukas, E., Gasteratos, A., and Visentin, G., “Towards orbital based global rover localization,” 2015 IEEE International Conference on Robotics and Automation (ICRA), 2015, pp. 2874-2881. doi:10.1109/ICRA.2015.7139591.

[10] Brauer, G., Cornick, D., and Stevenson, R., "Capabilities and Applications of the Program to Optimize Simulated Trajectories (POST). Program Summary Document,” 1977.

[11] Bellantoni, J., and Koenke, E., "Launch Pad Alignment of a Strapdown Navigator by the Kalman Filter," Control and Flight Dynamics Conference, 1968, p. 831.

[12] Lightsey, E. G., Mogensen, A. E., Burkhart, P. D., Ely, T. A., and Duncan, C., "Real-time navigation for Mars missions using the Mars network," Journal of Spacecraft and Rockets, Vol. 45, No. 3, 2008, pp. 519-533.

[13] Savage, P. G., "Strapdown Inertial Navigation Integration Algorithm Design Part 1: Attitude Algorithms," Journal of Guidance, Control, and Dynamics, Vol. 21, No. 1, 1998.

[14] Savage, P. G., "Strapdown Inertial Navigation Integration Algorithm Design Part 2: Velocity and Position Algorithms," Journal of Guidance, Control, and Dynamics, Vol. 21, No. 2, 1998.

[15] Rogers, R. M., Applied mathematics in integrated navigation systems, American Institute of Aeronautics and Astronautics, 2007.

[16] Honeywell-Aerospace, "HG9900 Specification Sheet," https://aerospace.honeywell.com, 2009.

[17] Norris, L., Tao, Y.-C., Hall, R., Chuang, J., and Whorton, M., "Analysis of Ares 1 Ascent Navigation Options," AIAA Guidance, Navigation and Control Conference and Exhibit, American Institute of Aeronautics and Astronautics, 2008.

[18] NorthropGrumman, "LN200S Specification Sheet," https://www.northropgrumman.com, 2013.

[19] Honeywell-Aerospace, "HG1930 Specification Sheet," https://aerospace.honeywell.com, 2016.

[20] Oliver Jr, T. E., "Navigation system design with application to the Ares I Crew Launch Vehicle and Space Launch Systems," Master's thesis, Mississippi State University, 2013.

[21] Patrick, S., Oliver, T. E., and Anzalone, E., "Assessment and Verification of SLS Block 1B Exploration Upper Stage and Stage Disposal Performance," Advances in the Astronautical Sciences: Guidance, Navigatino, and Control 2017, Vol. 159, American Astronautical Soceity, Univelt, 2017.

[22] Williams, J., Senent, J., Ocampo, C., and Lee, D. E., "Recent Improvements to the Copernicus Trajectory Design and Optimization System,” 22nd AAS/AIAA Space Flight Mechanics Meeting, 2012.

[23] Parish, J. J., Parish, A. S., Swanzy, M., Woodbury, D., Mortari, D., and Junkins, J. L., "The Stellar Positioning System (Part I): An Autonomous Position Determination Solution,” NASA-MSFC Report, Vol. 1, No. 1, 2009, pp. 1-30.

[24] Woodbury, D. P., Parish, J. J., Parish, A. S., Swanzy, M., Denton, R., Mortari, D., and Junkins, J. L., “The Stellar Positioning System (Part II): Improving Accuracy During Implementation,” NASA-MSFC Report, Vol. 1, No. 2, 2009, pp. 1-20.

[25] Dukeman, G., "Atmospheric Ascent Guidance for Rocket-Powered Launch Vehicles," AIAA Guidance, Navigation, and Control Conference and Exhibit, American Institute of Aeronautics and Astronautics, 2002. 